\title{
Efecto del Seguimiento a la Gestión Estratégica sobre las Funciones Sustantivas en las Instituciones de Educación Superior en Colombia*
}

\author{
Effect of Monitoring the Strategic Management on the Substantive Functions in Higher Education Institutions in \\ Colombia \\ Efeito do Monitoramento da Gestão Estratégica sobre as Funções Substantivas em Instituições de Ensino Superior \\ na Colômbia
}

Carlos Hernán González-Campo

Universidad del Valle, Colombia

ORCID: https://orcid.org/0000-0003-2120-8209

DOI: https://doi.org/10.11144/Javeriana.cao33.esge

Oscar Iván Vásquez-Rivera ${ }^{\mathrm{a}}$

Universidad Santiago de Cali, Colombia

oscar.vasquez04@usc.edu.co

ORCID: https://orcid.org/0000-0001-7867-2761

Recibido: 23 Febrero 2020

Aceptado: 29 Octubre 2020

Jairo Humberto Cifuentes Madrid

Pontificia Universidad Javeriana, Colombia

ORCID: https://orcid.org/0000-0003-3980-0403

Publicado: 20 Diciembre 2020

\section{Resumen:}

¿Cuál es el efecto que tiene el seguimiento a la gestión estratégica sobre las funciones sustantivas de investigación, docencia y extensión en las Instituciones de Educación Superior -IES- en Colombia? El objetivo de esta investigación es responder al interrogante, por medio del uso de una metodología cuantitativa sobre una muestra compuesta por 83 instituciones. La hipótesis plantea que el seguimiento a la gestión estratégica genera un efecto positivo en las funciones sustantivas. Los resultados respaldan el planteamiento, siendo significativo sólo para la docencia y la investigación. Esto permite concluir que las IES pueden centrar su gestión estratégica hacia las variables del seguimiento que lo componen, dado los impactos positivos de este proceso sobre las funciones sustantivas de docencia, investigación y extensión.

Códigos JEL: I23, M10.

Palabras clave: Seguimiento a la gestión estratégica, funciones sustantivas, Instituciones de Educación Superior, Colombia.

\section{Abstract:}

What is the effect of monitoring strategic management on the substantive functions of research, teaching and extension in Higher Education Institutions -HEI- in Colombia? The objective of this research is to answer the question, through the use of a quantitative methodology on a sample composed of 83 institutions. The hypothesis suggests that monitoring of strategic management generates a positive effect on substantive functions. The results support the approach, being significant only for teaching and research. This allows us to conclude that HEIs can focus their strategic management on the monitoring variables that compose it, given the positive impacts of this process on the substantive functions of teaching, research and extension.

JEL Codes: I23, M10.

Keywords: Monitoring strategic management, substantive functions, Higher Education Institutions, Colombia.

\section{Resumo:}

Qual é o efeito do monitoramento da gestão estratégica nas funções substantivas de pesquisa, ensino e extensão em Instituições de Ensino Superior -IES- na Colômbia? O objetivo desta pesquisa é responder ao questionamento, por meio do uso de uma metodologia quantitativa em uma amostra composta por 83 instituições. A hipótese propõe que o monitoramento da gestão estratégica gera um efeito positivo nas funções substantivas. Os resultados sustentam a abordagem, sendo significativos apenas para ensino e pesquisa. Isso permite concluir que as IES podem focar sua gestão estratégica nas variáveis de monitoramento que o compõem, dados os impactos positivos desse processo nas funções substantivas de ensino, pesquisa e extensão.

Notas de autor

\footnotetext{
a Autor de correspondencia. Correo electrónico: oscar.vasquez04@usc.edu.co
} 
Códigos JEL: I23, M10.

Palavras-chave: Monitoramento da gestão estratégica, funções substantivas, Instituições de Ensino Superior, Colombia.

\section{Introducción}

El estudio realizado por la red Telescopi Colombia en el 2011, cuyo objetivo general era conocer y compartir las experiencias en los procesos de planeación de las universidades, permitió determinar que el seguimiento a la gestión estratégica era lo más importante y relevante a reconocer para la dirección universitaria, debido a que la mayoría de Instituciones de Educación Superior -IES - tanto públicas como privadas, contaban con una cultura sólida de elaboración de sistemas de planeación y sus prioridades giraban en torno a medir, evaluar, verificar y controlar la ejecución de la estrategia institucional.

En ese contexto, uno de los principales interrogantes que surgió era ¿cuáles son los componentes del seguimiento a la gestión estratégica que tienen efecto positivo en las funciones sustantivas de las IES (MEN, 2007)? Dicho interrogante deja a su paso conclusiones importantes: en primera instancia, existen pocas investigaciones nacionales e internacionales en las que se pueda evidenciar claramente dichos componentes y sus efectos (Álvarez, Tortosa \& Pellín, 2015; Cáceres, 2007; Concepción \& Trujillo, 2014; Cook, Nelson, \& Lillis, 2013; De Dios-Alija, García, \& Muñoz-Pérez, 2017; Del Huerto, 2007; Llinàs-Audet, Girotto \& Parellada, 2011; López, Sánchez, Pardo \& Zapata, 2017; Parakhina, Godina, Boris \& Ushvitsky, 2017; Sánchez-del-Arco \& Peón, 2017); en segunda instancia, es importante indicar que la mayoría de estudios tienen enfoque metodológico cualitativo, lo que dificulta evidenciar de donde proviene el impacto positivo en las funciones sustantivas de las IES y, de igual forma, determinar aquellas características que influyan en la dirección del impacto entre las variables dependientes e independientes.

Adicionalmente, los estudios presentan diferentes orientaciones conceptuales, por lo tanto, no todas coinciden en la determinación de las variables del seguimiento a la gestión estratégica que tienen mayor efecto positivo sobre las funciones sustantivas en las IES. En ese sentido, esta investigación parte de la construcción de una variable independiente denominada Seguimiento a la Gestión Estratégica compuesta por cuatro componentes de evaluación teniendo en cuenta la información teórica revisada. Posteriormente, se realiza un análisis de componentes principales a partir de la valoración de los mismos a través de una encuesta distribuida virtualmente a 83 instituciones de las IES a nivel nacional. Esto permitirá realizar el análisis estadístico con las variables dependientes definidas a partir de las tres funciones sustantivas en las IES en Colombia para identificar su relación causal.

Este artículo muestra, en su primera parte, la revisión de la literatura bajo la cual se define el seguimiento a la gestión estratégica en las IES y se resaltan los estudios que muestran las variables del seguimiento a la gestión estratégica que tienen efecto positivo sobre tres funciones sustantivas. En la segunda parte, se expone la metodología, de índole cuantitativa, y la cual incluye una descripción de la muestra y las variables seleccionadas. Por último, en la tercera parte, se evidencian los resultados obtenidos a través de una regresión binaria tipo Probit y las principales conclusiones, dando un orden de logro de objetivos y resultados obtenidos.

\section{Marco teórico}

\section{El seguimiento a la Gestión Estratégica en las IES}

Iterando la necesidad de caracterizar el concepto de seguimiento a la gestión estratégica con el fin de mostrar el impacto que tiene en las funciones sustantivas de las IES, el seguimiento a la gestión estratégica se entiende según Velandia y Girotto (2015) como el proceso de aprendizaje y mejora continua que se da en la institución, 
con base en la revisión y evaluación de la estrategia elegida, unido al apoyo de la información confiable y oportuna con el fin de tomar decisiones transparentes y basadas en la objetividad. También recoge el establecimiento de mecanismos pertinentes para supervisar y realizar una evaluación periódica de cómo se desarrolla la estrategia y garantizando instrumentos para realizar rendiciones de cuentas.

En este sentido, la estrategia elegida de la cual hace mención Velandia y Girotto (2015) se encuentra documentada en cada institución y es conocida como Plan Estratégico; que es la hoja de ruta para alcanzar la misión y visión declarada por cada una de ellas. En él, los objetivos, los medios para alcanzarlos y las herramientas que se usarán para medir su progreso deben estar claramente definidos. El seguimiento del Plan Estratégico es fundamental, ya que permite evaluar y medir el estado de los procesos, subprocesos y cada una de las actividades que se encuentran dentro de ellas, para tomar decisiones preventivas, regulativas o correctivas que eviten desviaciones en el plan o que corrija los errores presentados. A la vez, el seguimiento debe permitir a los ejecutores del Plan Estratégico establecer la brecha entre el estado actual del plan y de los objetivos propuestos.

Para efectos de la investigación, es de interés particular conocer el estado actual del plan estratégico, como consecuencia que tan solo es posible conocer las expectativas de las IES que ya hayan elaborado su plan estratégico. Aún más determinante es conocer la manera en la cual cada una de las variables de seguimiento ha impactado a las tres funciones sustantivas, para lo cual sería indispensable que las IES estén ejecutando el plan estratégico formulado.

Teóricamente se ha abordado el seguimiento a la gestión estratégica en las IES desde diversas perspectivas, una es la que la considera como un sistema de gestión. De acuerdo con Ferreira y Otley (2009), estos los sistemas se definen de la siguiente manera:

Son los mecanismos, procesos, sistemas y redes formales e informales que evolucionan, utilizados por las IES para transmitir los objetivos y metas claves definidos por la administración, para ayudar al proceso estratégico y la gestión continua a través del análisis, planificación, medición y control. En general, apoyan y facilitan el aprendizaje organizacional y el cambio (Ferreira \& Otley, 2009, p. 264).

El concepto aún sigue evolucionando y los investigadores han determinado que los sistemas de comunicación entre las unidades organizacionales de las IES y sus características financieras son determinantes a la hora de definir el plan estratégico (Broadbent \& Laughlin, 2009; Chenhall, 2003; Chenhall \& Euske, 2007; Chenhall, Hall, \& Smith, 2010; Ferreira \& Otley, 2009; Otley, 2003); estas se encuentran dentro de las características de los controles, que incluyen herramientas formales de planificación, como el uso de presupuestos y metas para establecer expectativas y contra las cuales monitorear el desempeño, que son "deliberadamente articulados" (Chenhall et al., 2010, p. 742) para la toma de decisiones. Los controles informales se caracterizan por el uso de información y procesos más fluidos utilizando el diálogo y la comunicación como las principales formas de compartir información sobre las actividades y el desempeño.

De acuerdo con Deem (1998), Deem y Brehony (2005) y Aspromourgos (2012) los sistemas de gestión de las IES, en la actualidad, se asemejan a las de cualquier empresa del sector privado, dado que, como cualquier otro tipo de organización que elabora productos básicos, se debe dar respuesta a determinados grupos de interés. En este caso particular (en los servicios de enseñanza y de investigación) se requiere que las IES respondan a sus "clientes", incluyendo estudiantes, empleadores, consejos de investigación y consejos que las financian (Willmott, 1995; Parker, 2002, 2013). Broadbent, Gallop y Laughlin (2010) exponen esta situación al ejemplificar como las entidades gubernamentales condicionan la entrega de recursos a las IES a la consecución de resultados. Esas condiciones generan presión interna en el seguimiento de la estrategia en los departamentos internos de las IES, los cuales deben contribuir al alcance de las metas propuestas.

Desde tal perspectiva, es importante tener el conocimiento de las características que abarcan las instituciones que hacen parte del estudio, pues de acuerdo con ellas es posible conocer con mayor detalle los servicios que ofrecen, su estructura, su funcionalidad, sus recursos, sus grupos de interés y las necesidades y 
requerimientos a los cuales deben responder, y de cuya incidencia se desprenden respuestas distintas sobre las tres funciones sustantivas.

Otra perspectiva sobre el seguimiento a la gestión estratégica en las IES es aquella que la considera como un proceso o método de monitoreo, evaluación y aseguramiento de la calidad de la estrategia (Centro Interuniversitario de Desarrollo, 1992; 1999; 2007). Esta perspectiva incluye el desarrollo de políticas de seguimiento, indicadores (v.gr. indicadores de competitividad, estratégicos, tácticos, operativos, de desempeño, de rendimiento, de resultado y de gestión), herramientas (v.gr. cuadros de mando, elaboración de memorias anuales, información estadística, dashboard, evaluación por pares, revisión por autoridades externas, rendición de cuentas), cuyo objetivo es retroalimentar a los directivos de las IES con información de la institución que permita mejorar la toma de decisiones constante y reiterada, con la convicción que siempre es posible mejorar. El equipo de investigación reitera su importancia, como la razón por la cual es posible conocer de qué manera cada una de las variables del estudio impactan la gestión de las tres funciones sustantivas, y por ende, su resultado parcial y final.

El Centro Interuniversitario de Desarrollo $(1992 ; 1999 ; 2007)$ agrega que desde la fase de planificación hay que prever al método de seguimiento: responsabilidades, frecuencias, muestras, etc. Esta es una de las acciones importantes de la fase de ejecución, que permite descubrir y corregir desajustes en la inmediatez, a diferencia de la fase de evaluación, que se lleva a cabo al final del período determinado y que sirve para tomar decisiones para el período siguiente.

En este contexto, las dos perspectivas teóricas presentadas no son antagónicas, inclusive, en muchos casos, son utilizadas indistintamente por las IES para la realización del seguimiento a la gestión estratégica. El desarrollo de esta investigación se enfoca en considerar el seguimiento a la gestión estratégica en las IES desde la perspectiva del proceso o método.

La importancia del desarrollo de planes estratégicos dentro de las IES se ha convertido en tema central a lo largo del siglo XXI. Keller (2007), Bowen y Schultz citados por Keller (2007), así como García y Palomares (2009) descubrieron que los directivos han puesto sus esfuerzos en mejorar la calidad de las instituciones de educación porque son conscientes de que el mejoramiento en la calidad del sector implica el mejoramiento de su capital humano, el cual impulsa el crecimiento económico, la seguridad nacional, la salud pública, la sagacidad política y la conservación de las culturas existentes, así como el libre desarrollo y expresión de las nuevas culturas. Además, los actores externos del entorno presionan constantemente a las IES para utilizar los recursos más eficientemente, y un claro ejemplo del caso son las exigencias de la OCDE en el sector de la educación.

Por otra parte, de acuerdo con Rivero, Díaz, López y Rodríguez (2018), los gobiernos impulsan la generación de conocimientos y habilidades desde las IES con el fin de efectuar un impacto positivo sobre el desarrollo económico y social en sus naciones. Esta perspectiva hace que se centre la atención sobre la forma en la que las IES están midiendo y mejorando sus resultados tanto operativos como estratégicos, aspecto en el que varios autores han ofrecido diferentes factores críticos que se pueden valorar para reconocer aquel efecto (Keller, 2007; García \& Palomares, 2009; Rivero et al., 2018). Entre los muchos factores críticos que se han analizado, hay tres que son sobresalen por ser determinantes en la calidad de las IES: la docencia, la investigación y la extensión.

También es de interés, para la presente investigación, analizar cuál es el impacto que reciben estas funciones a partir del seguimiento que se realiza a la gestión estratégica en las IES. Pese a que los estudios citados convergen en que la naturaleza cambiante del entorno externo de las instituciones hace que sea difícil medir, gestionar y controlar las tres funciones, la comprensión de la incidencia entre el seguimiento a la gestión estratégica sobre el mejoramiento de cada una de ellas, les permitirá a los directivos de todas las IES enfocar sus esfuerzos administrativos en aspectos que realmente conduzcan a fortalecer su naturaleza y de esta manera contribuir al propósito de generar conocimiento que impacte a la sociedad. 


\section{Hipótesis}

A partir de la revisión de la literatura y de acuerdo con el objetivo de investigación se plantean las siguientes hipótesis:

$\mathrm{H}_{1}$ : El seguimiento a la gestión estratégica afecta positivamente la probabilidad de mejoría de la función sustantiva de docencia.

$\mathrm{H}_{2}$ : El seguimiento a la gestión estratégica afecta positivamente la probabilidad de mejoría de la función sustantiva de investigación.

$\mathrm{H}_{3}$ : El seguimiento a la gestión estratégica afecta positivamente la probabilidad de mejoría de la función sustantiva de extensión.

\section{Metodología}

La metodología de esta investigación es de enfoque cuantitativo y de tipo explicativa. Con el fin de estudiar el efecto del seguimiento a la gestión estratégica sobre las tres funciones sustantivas de las IES.

\section{Muestra}

La encuesta inició su aplicación el 1 de agosto de 2016 y se cerró el 21 de septiembre del mismo año, el cuestionario fue enviado por correo electrónico a las rectorías de las 288 instituciones educativas mediante el aplicativo web SurveyGizmo. En total, respondieron 85 rectorías de IES en Colombia sobre una población total de 288 (30\%). Se definió una muestra de 164 respuestas teniendo en cuenta un 95\% de nivel de confianza y $5 \%$ de error. Finalmente, se obtuvo una tasa de respuesta del 51,8\%, sin embargo, debido a que la encuesta se aplicó vía web, se tuvo en consideración un estudio sobre la tasa de respuesta a través de Internet, donde se considera que entre un $25 \%$ y $30 \%$ es representativo para el universo de interés, lo que nos permite establecer que la respuesta de 85 rectorías satisface las condiciones estadísticas de significancia (Cook, Heath, \& Thompson, 2000). Ahora bien, el análisis se realiza con 83 respuestas debido a que de las 85 rectorías que contestaron la encuesta, 75 indicaron que tienen su plan estratégico en plena operación, el resto indicaron que están pendientes de iniciar la implementación o están en elaboración del plan, solo dos rectorías manifestaron que no existe plan estratégico. Estos últimos fueron excluidos del análisis para concentrarse en los datos sobre los cuales existe un plan estratégico en el cual se pueda tener noción del seguimiento.

\section{Variables}

Para contrastar las hipótesis planteadas en esta investigación se realiza una regresión binaria tipo Probit que incluye como variables dependientes las funciones sustantivas y como variable independiente el seguimiento a la gestión estratégica; además, se utilizan variables de control como son: tipo de IES y estado del plan estratégico.

\section{Dependientes}

1. Función Sustantiva de Docencia: hace referencia a si ha mejorado la función sustantiva de Docencia debido a la planeación estratégica. Para esta investigación la variable es dicotómica: toma el valor de 1 si ha mejorado, y 0 en caso contrario. 
De acuerdo con Alonso (2019) y Morán (2004) el docente, más allá de la transmisión de conocimientos, concientiza a los estudiantes sobre la realidad de su profesión, con herramientas que le brindan nociones básicas y que despierten su propio interés por investigar, reeducarse y participar en los procesos de actualización de la información de su área de conocimiento, generando así un aporte propio a la profesión.

El rol del docente como guía y mentor juega un papel muy importante, dado que la limitación del significado de la docencia a una mera tarea de transmisión de información implicaría ignorar los procesos de aprendizaje individuales de los estudiantes, que varían de acuerdo con las características de cada persona.

¿Cómo se podría medir la calidad de esta función en una IES? En el contexto colombiano, uno de los indicadores más utilizados para medir la calidad de esta función sustantiva, consiste en la cuantificación de la cantidad de docentes que cuentan con títulos de posgrado, indicador que funge como evidencia de la educación continua del profesorado de cada IES.

En conclusión, la docencia es una labor bidireccional entre el estudiante y su formador, pues mientras el docente transmite el conocimiento y evalúa el aprendizaje, el alumno deberá formar un criterio autónomo acerca de la profesión, y utilizar al docente como un guía de formación de sus conceptos.

2. Función Sustantiva de Investigación: hace referencia a si ha mejorado la función sustantiva de Investigación debido a la planeación estratégica. Para esta investigación la variable es dicotómica: toma el valor de 1 si ha mejorado, y 0 en caso contrario.

La investigación se refiere al conocimiento que las IES producen, y según Canales (2011) se trata de un concepto que ha ido cambiando durante las últimas décadas. Inicialmente era una herramienta que se usaba exclusivamente con fines económicos y lucrativos, y que impartía conocimientos útiles exclusivamente a los sectores productivos de cada país. Posteriormente, se tomaron en cuenta los beneficios sociales y medioambientales que se podían obtener de los conocimientos, al conocer las realidades y necesidades que se viven en las distintas comunidades y que podrían hacer parte de los objetivos de los planes de desarrollo de cada país.

En la investigación de González y Arcángel (2013), es posible identificar un dilema que rodea este concepto, el cual consiste en la relación entre las funciones sustantivas de investigación y docencia. Este dilema parte de la idea de que, en la medida que el docente esté actualizado en la realidad de su profesión, les entregará conocimientos de mejor calidad a sus estudiantes. Adicionalmente, al tomar en cuenta y perfeccionar las indagaciones que sus mismos alumnos han realizado, para incluirlos en sus propias investigaciones, estaría motivando a sus estudiantes a ser personas curiosas que deseen profundizar su conocimiento por medio de la investigación.

En el contexto latinoamericano, y de acuerdo con Rivero et al. (2018), esta función es medida con frecuencia según la cantidad de publicaciones en revistas científicas, participación en proyectos de investigación, participación en eventos científicos, cantidad de patentes y de registros.

3. Función Sustantiva de Extensión: hace referencia a si ha mejorado la función sustantiva de Extensión debido a la planeación estratégica. Para esta investigación la variable es dicotómica: toma el valor de 1 si ha mejorado, y 0 en caso contrario.

Partiendo desde las ideas convergentes de la Universidad del Valle (2012) y la Universidad del Bosque (2014), es posible determinar que la función de extensión hace parte de la proyección social de cada IES. La proyección social es la forma en la que las actividades de las instituciones (investigación, docencia, desarrollo de tecnologías, productos y procesos, adquisición de nuevos conocimientos por parte de docentes y estudiantes) aportan a la resolución de problemas sociales, culturales, económicos, de salud pública, tecnológicos, etc., bien sea a nivel local, regional, nacional o internacional. El desarrollo en competencias y conocimientos integrales que cada institución le ofrezca a sus integrantes será determinante para educar a personas que generen proyectos de mayor impacto para su entorno. 


\section{Independientes}

El Seguimiento a la Gestión Estratégica se definió como variable, obteniéndose del promedio de las cuatro variables principales sobre las cuales se compone el proceso de seguimiento a la gestión estratégica, teniendo en cuenta la revisión de la literatura y de considerarla desde la perspectiva del proceso o método:

1. Monitoreo y Evaluación: variable dicotómica que toma el valor de 1 si la IES realiza monitoreo y evaluación formal del plan estratégico, y 0 en caso contrario.

2. Resultados de Autoevaluación: variable dicotómica que toma el valor de 1 si la IES en el proceso de planificación estratégica tiene en cuenta los resultados de la autoevaluación de la institución y del plan estratégico anterior, y 0 en caso contrario.

3. Sistema de Indicadores: variable dicotómica que toma el valor de 1 si la IES dispone de un sistema de indicadores diseñado específicamente para el seguimiento estratégico, y 0 en caso contrario.

4. Revisión en Unidades Internas: Variable dicotómica que toma el valor de 1 si la IES cuenta con una revisión periódica del plan estratégico en relación con su despliegue y los resultados dentro de sus unidades internas, y 0 en caso contrario.

TABLA 1

Definición, impacto y ejemplo justificado de la variable independiente Monitoreo y Evaluación

\begin{tabular}{|c|c|c|}
\hline \multicolumn{3}{|c|}{ Monitoreo y Evaluación } \\
\hline Definición & Impacto & Ejemplo \\
\hline $\begin{array}{l}\text { Velandia y Girotto (2015), así } \\
\text { como De Waal y Kerklaan (2015) } \\
\text { lo determinaron como una } \\
\text { herramienta, que permite } \\
\text { establecer pautas de acción para } \\
\text { alcanzar un objetivo específico, } \\
\text { teniendo en cuenta factores } \\
\text { internos y externos de la } \\
\text { organizacióny sus unidades. } \\
\text { La finalidad de este } \\
\text { procedimiento consiste en } \\
\text { identificar si las acciones } \\
\text { ejecutadas están contribuyendo al } \\
\text { alcance de los objetivos } \\
\text { propuestos, o si es necesario } \\
\text { realizar ajustes, cambios o } \\
\text { correcciones. }\end{array}$ & $\begin{array}{l}\text { De acuerdo con la investigación } \\
\text { ejecutada por, Ramirez-Gutiérrez, } \\
\text { Barrachina-Palanca y Ripoll-Feliu } \\
\text { (2020)en universidades públicas } \\
\text { de Colombia y España, el uso } \\
\text { eficiente de los recursos fisicos y } \\
\text { humanos (personal de trabajo, } \\
\text { infraestructura, tecnologia, gastos } \\
\text { operativos) han incidido } \\
\text { directamente en las funciones de } \\
\text { docencia e investigación; por lo } \\
\text { tanto, el monitoreo y la } \\
\text { evaluación de la disposición de } \\
\text { estos recursos dentrode las } \\
\text { universidades sondeterminantes } \\
\text { para generar un impacto positivo } \\
\text { en esas funciones sustantivas. }\end{array}$ & $\begin{array}{l}\text { De Waal y Kerklaan (2015) } \\
\text { propusieron el Balance } \\
\text { Scorecard como una de las } \\
\text { herramientas más integrales } \\
\text { para proponer objetivosy } \\
\text { acciones explicitas que les } \\
\text { permita alcanzarlas. } \\
\text { Enfatizan, especialmente, en } \\
\text { que permite tomar en } \\
\text { consideración el impacto de } \\
\text { los factores externos a la } \\
\text { organización, los cuales no } \\
\text { están bajo su control. }\end{array}$ \\
\hline
\end{tabular}

Fuente: elaboración propia.

TABLA 2

Definición, impacto y ejemplo justificado de la variable independiente Resultados de Autoevaluación

\begin{tabular}{|c|c|c|}
\hline \multicolumn{3}{|c|}{ Resultados de Autoevaluación } \\
\hline Definición & Impacto & Ejemplo \\
\hline $\begin{array}{l}\text { De acuerdo con Kniola (2013), } \\
\text { Shah \& Nair (2011), la } \\
\text { autoevaluación debe conducira a } \\
\text { una reflexión objetiva, quele } \\
\text { permita a cada unidad } \\
\text { organizacional identificar cuáles } \\
\text { de sus labores generan aportes a } \\
\text { las tareas críticas de la } \\
\text { organización, para } \\
\text { posteriormente evaluar si las } \\
\text { acciones que están tomando son } \\
\text { las más acordes para generar los } \\
\text { resultados esperados. }\end{array}$ & $\begin{array}{l}\text { En el caso de las IES de Colombia, estas } \\
\text { tienden a buscar reconocimiento de sus } \\
\text { funciones sustantivas por medio del } \\
\text { Sistema Nacional de Acreditación, el } \\
\text { cual establece dentro de sus } \\
\text { procedimientos y condiciones, que para } \\
\text { que un programa pueda ser candidato a } \\
\text { una acreditación, deberá realizar un } \\
\text { proceso de autoevaluación, que incluye } \\
\text { factores como la calidad de la educación } \\
\text { que ofrece, las redes de investigación en } \\
\text { las que participa, y la proyección social } \\
\text { que genera; todo de acuerdo con el } \\
\text { articulo } 3 \text { del Acuerdo } 02 \text { de } 2012 \text {. }\end{array}$ & $\begin{array}{l}\text { Kniola (2013), Shah \& Nair } \\
\text { (2011) realizaron estudios en } \\
\text { Estados Unidos y Australia, en } \\
\text { donde analizaron como la auto } \\
\text { elaboración del sistema de } \\
\text { evaluación permitió a los } \\
\text { departamentos administrativos de } \\
\text { las IES encontrar deficiencias en } \\
\text { su estructuración, la elaboración } \\
\text { de ese sistema permitióevalúa la } \\
\text { eficiencia y eficacia de la } \\
\text { comunicación entre los miembros } \\
\text { de los departamentos que fueron } \\
\text { objeto de estudio. }\end{array}$ \\
\hline
\end{tabular}

Fuente: elaboración propia. 
TABLA 3

Definición, impacto y ejemplo justificado de la variable independiente Sistema de Indicadores

\begin{tabular}{|c|c|c|}
\hline \multicolumn{3}{|c|}{ Sistema de Indicadores } \\
\hline Definición & Impacto & Ejemplo \\
\hline $\begin{array}{l}\text { Para Murias, de Miguel y } \\
\text { Rodriguez (2007), un } \\
\text { sistema de indicadores } \\
\text { consiste en la construcción } \\
\text { de unidades de medida bajo } \\
\text { las que se determina la } \\
\text { consecución de los } \\
\text { objetivos propuestos, y los } \\
\text { cuales dependen del } \\
\text { sistema de valoración de } \\
\text { cada IES, así como de sus } \\
\text { caracteristicas internas y } \\
\text { externas. }\end{array}$ & $\begin{array}{l}\text { El impacto de los sistemas de } \\
\text { indicadores de las IES en cada una } \\
\text { de las variables dependientes } \\
\text { (docencia, investigacióny } \\
\text { extensión) no solo dependerá de la } \\
\text { herramienta que se seleccione para } \\
\text { medir su eficiencia, sino también de } \\
\text { las tareas que se consideren criticas, } \\
\text { las unidades a las que pertenecen } \\
\text { esas labores, y el impacto que estas } \\
\text { puedan generar en otros } \\
\text { departamentos. Navas et al. (2020) } \\
\text { partieron de la base que existia tal } \\
\text { relación entre la función sustantiva } \\
\text { de investigación y la eficiencia de } \\
\text { las universidades que hicieron parte } \\
\text { de su estudio. Asi, se puede inferir } \\
\text { que profesores que desarrollen } \\
\text { actividades de investigación } \\
\text { (participen en grupos de } \\
\text { investigación, que realicen } \\
\text { maestriaso doctorados, etc.) } \\
\text { puedan ofrecer conocimientos que } \\
\text { reflejen la realidad de la profesión. }\end{array}$ & $\begin{array}{l}\text { En la investigación } \\
\text { desarrollada por Navas et al. } \\
\text { (2020), cuyo objetivo final } \\
\text { era evaluar la eficiencia de } \\
\text { las IES de Colombia, } \\
\text { tomando en cuenta las } \\
\text { métricas que el sistema } \\
\text { educativo del país } \\
\text { considerara importantes; los } \\
\text { investigadores determinaron } \\
\text { que uno de los indicadores } \\
\text { más comunes para medir la } \\
\text { calidad de la docencia en } \\
\text { Colombia estaba } \\
\text { directamente relacionado } \\
\text { con el porcentaje de } \\
\text { profesores que contaran con } \\
\text { posgrados (maestrías y } \\
\text { doctorados). }\end{array}$ \\
\hline
\end{tabular}

Fuente: elaboración propia.

TABLA 4

Definición, impacto y ejemplo justificado de la variable independiente Revisión de Unidades Internas

\begin{tabular}{|c|c|c|}
\hline \multicolumn{3}{|c|}{ Revisión en Unidades Internas } \\
\hline Definición & Impacto & Ejemplo \\
\hline $\begin{array}{l}\text { De Waal \& Kerklaan (2015) } \\
\text { afirman que todos los objetivos } \\
\text { estratégicos a nivel } \\
\text { organizacional se traducen en } \\
\text { metas especificas para cada una } \\
\text { de sus unidades. Se convierteen } \\
\text { la tarea de los miembros de } \\
\text { cada unidadde la IES (bien sea } \\
\text { por facultades, departamentos u } \\
\text { oficinas) establecer las tareas } \\
\text { críticas por desarrollar, } \\
\text { determinar los indicadores para } \\
\text { medir el grado de desarrollo de } \\
\text { los procesos y determinar de } \\
\text { qué forma cada objetivo } \\
\text { unitario contribuye a la } \\
\text { consecución de la estrategia } \\
\text { institucional. }\end{array}$ & $\begin{array}{l}\text { Además del establecimiento } \\
\text { de tareas criticas y creación } \\
\text { de indicadores, la revisión de } \\
\text { unidades internas permite la } \\
\text { cohesión de las unidades } \\
\text { institucionales. Tal es el caso } \\
\text { de la Universidad Nacional } \\
\text { de Colombia (2005), que en } \\
\text { sus Estatutos han } \\
\text { determinado los principios } \\
\text { básicos que cada uno de sus } \\
\text { departamentos deberán } \\
\text { cumplir, y que permitanla } \\
\text { alineación de sus objetivos } \\
\text { con los del resto de } \\
\text { unidades. }\end{array}$ & $\begin{array}{l}\text { En un estudio realizadopor } \\
\text { Meyer Junior, Pascuci \& } \\
\text { Meyer (2018), fue posible } \\
\text { encontrar que los directivos de } \\
\text { nivel intermedio } \\
\text { frecuentemente juegan un } \\
\text { papel relevante en la } \\
\text { conciliación entre los intereses } \\
\text { de los directivos de nivel } \\
\text { superior, con los de otras } \\
\text { unidades académicas que se } \\
\text { puedan contraponer a ellos. } \\
\text { Desenfocando la atención a } \\
\text { los intereses individuales y } \\
\text { enfocándolo en los que son } \\
\text { comunes a todos. }\end{array}$ \\
\hline
\end{tabular}

Fuente: elaboración propia.

TABLA 5

Fiabilidad y validez: variable seguimiento a la gestión estratégica

\begin{tabular}{|c|c|c|c|c|c|c|}
\hline \multicolumn{7}{|c|}{$\begin{array}{l}\text { 1) Monitoreo y Evaluación, 2) Resultados Autoevaluación, } \\
\text { 3) Sistema de Indicadores, 4) Revisión en Unidades Internas. }\end{array}$} \\
\hline $\begin{array}{l}\text { Alfa de } \\
\text { Cronbach }\end{array}$ & Factorial & $\begin{array}{l}\text { Explicación de } \\
\text { la varianza }\end{array}$ & $\begin{array}{l}\text { Sig. de } \\
\text { Bartlett }\end{array}$ & $\mathrm{KMO}$ & Media & $\begin{array}{c}\text { Desviación } \\
\text { tipica }\end{array}$ \\
\hline 0,789 & 1 & 0,6396 & 0,000 & 0,66 & 0,9217 & 0,20999 \\
\hline
\end{tabular}

Fuente: elaboración propia.

El alfa toma valores entre 0 a 1 , donde cuanto más cercano esté a la unidad mayor consistencia tendrán los componentes entre sí. En el contexto de estudio se puede concluir que los componentes son consistentes entre sí de acuerdo con el alfa; luego, se realizan las pruebas de significancia de Bartlett y el coeficiente KMO que indican si para el conjunto de datos es consistente o factible realizar el análisis factorial. La prueba de significancia de Bartlett contrasta la hipótesis nula que la matriz de correlaciones es una matriz identidad, lo que sugiere que no hay correlaciones significativas en el conjunto de datos y, por lo tanto, no sería correcto realizar un análisis factorial al conjunto de datos. En el contexto propio se rechaza la hipótesis nula, por lo cual sería apropiado hacer un análisis factorial; por otro lado, la prueba KMO (Kaiser-Meyer-Olkin) contrasta qué tan pequeñas son las correlaciones parciales de las variables, al igual que el alfa de Cronbach se mueve en 
una escala de 0 a 1 , donde los resultados cercanos a uno indican que es apropiado realizar un análisis factorial; por lo anterior, se concluye que es apropiado realizar un análisis factorial al conjunto de datos.

\section{Variables de Control}

Tipo de IES: variable categórica que es igual a 1 si la IES es Institución Universitaria/Escuela Tecnológica, Institución Tecnológica, Institución Técnica Profesional, y 0 si es Universidad. Se incluye esta variable para identificar si el tipo de institución diferente a universidad tiene incidencia significativa sobre las tres funciones sustantivas.

Desde la perspectiva legal en Colombia y la normatividad que la regula, todas las Instituciones de Educación Superior tienen como objetivo "profundizar en la formación integral de los colombianos dentro de las modalidades y calidades de la Educación Superior, capacitándolos para cumplir las funciones profesionales, investigativas y de servicio social que requiere el país" (Ley 30, 1992).

No obstante, la misma norma discrimina las entidades que ofrecen estos servicios en tres categorías: las Instituciones Técnicas Profesionales, las Universidades y, por último, las Instituciones Universitarias, también llamadas Escuelas Tecnológicas. Partiendo de esa base, se convierte en algo fundamental e importante definir con certeza la diferencia entre los tres tipos de instituciones; a continuación se presentará su definición de acuerdo con el marco legal colombiano:

TABLA 6

Categorización de las Instituciones de Educación Superior (IES) en Colombia

\begin{tabular}{|c|c|c|}
\hline Instituciones Técnicas Profesionales & Instituciones Tecnológicas & Universidades \\
\hline $\begin{array}{l}\text { Son Instituciones de Educación } \\
\text { Superior, que se caracterizan por su } \\
\text { vocación e identidad manifiesta en los } \\
\text { campos de los conocimientos y el } \\
\text { trabajo en actividades de carácter } \\
\text { técnico, debidamente fundamentadas en } \\
\text { la naturaleza de un saber, cuya } \\
\text { formación debe garantizar la } \\
\text { interacción de lo intelectual con lo } \\
\text { instrumental, lo operacional y el saber } \\
\text { técnico. }\end{array}$ & $\begin{array}{l}\text { Son Instituciones de } \\
\text { Educación Superior, que se } \\
\text { caracterizan por su vocación } \\
\text { e identidad manifiestas en los } \\
\text { campos de los conocimientos } \\
\text { y profesiones de carácter } \\
\text { tecnológico, con } \\
\text { fundamentación científica e } \\
\text { investigativa }\end{array}$ & $\begin{array}{l}\text { Son universidades las reconocidas } \\
\text { como tales y las instituciones que } \\
\text { acrediten su desempeño con criterio } \\
\text { de universalidad en las siguientes } \\
\text { actividades: la investigación } \\
\text { científica o tecnológica; la formación } \\
\text { académica en profesiones o } \\
\text { disciplinas y la producción, } \\
\text { desarrollo y transmisión del } \\
\text { conocimiento y de la cultura } \\
\text { universaly nacional. }\end{array}$ \\
\hline
\end{tabular}

Fuente: Ley 30 de 1992 y la Ley 749 de 2002.

En este sentido, partiendo de la definición de los tres tipos de IES, resulta de vital importancia resaltar los siguientes aspectos: primero, para el caso de los tres tipos de instituciones, los servicios educativos ofertados deben partir de un saber o conocimiento, el cual también debe contar con respaldo científico e investigativo en el caso de las universidades e instituciones tecnológicas. No obstante, dentro del marco legal, las universidades están sujetas a realizar actividades de investigación, y ejercer mecanismos de transmisión de conocimiento hacia su entorno.

Estas observaciones son importantes para la presente investigación, pues dependiendo de la clasificación cada una de las IES participantes, se podrá visualizar una mayor o menor cantidad de obligaciones y libertades para ejercer las funciones que se describen en las variables dependientes e independientes del presente estudio.

Estado del Plan Estratégico: variable categórica que toma el valor de 1 si el plan estratégico está en plena operación y 0 en cualquier otro caso (en elaboración o pendiente de iniciar implementación), se incluye esta variable para identificar si tener en plena operación el plan estratégico tiene incidencia significativa sobre las tres funciones sustantivas. 


\section{Resultados}

En esta sección se presentan los resultados del modelo y los estadísticos descriptivos. Para empezar, en la tabla 7 se muestran los estadísticos descriptivos de las variables dependientes e independientes. Se evidencia que el tipo de IES que en mayor cantidad contestó la encuesta son Instituciones Universitarias/Escuela Tecnológica, Institución Tecnológica, Institución Técnica Profesional con un 55\%. Adicionalmente, el 90\% de las IES tiene su plan estratégico en plena operación. Finalmente, es importante indicar que la mayoría de los encuestados indicó que las tres funciones sustantivas de las IES se ven mejoradas por el seguimiento a la gestión estratégica (docencia y extensión en 94\% e investigación en 93\%).

TABLA 7

Estadísticos descriptivos variables dependientes e independientes

\begin{tabular}{|c|c|c|c|}
\hline Variable & Alternativa & Frecuencia & $(\%)$ \\
\hline \multirow{2}{*}{$\begin{array}{l}\text { Función Sustantiva } \\
\text { de Docencia }\end{array}$} & $\begin{array}{l}\text { La planeación si ha servido para } \\
\text { mejorarla }\end{array}$ & 78 & $94 \%$ \\
\hline & $\begin{array}{l}\text { La planeación no ha servido } \\
\text { para mejorarla }\end{array}$ & 5 & $6 \%$ \\
\hline \multirow{2}{*}{$\begin{array}{l}\text { Función Sustantiva } \\
\text { de Investigación }\end{array}$} & $\begin{array}{l}\text { La planeación sí ha servido para } \\
\text { mejorarla }\end{array}$ & 77 & $93 \%$ \\
\hline & $\begin{array}{l}\text { La planeación no ha servido } \\
\text { para mejorarla }\end{array}$ & 6 & $7 \%$ \\
\hline \multirow{2}{*}{$\begin{array}{l}\text { Función Sustantiva } \\
\text { de Extensión }\end{array}$} & $\begin{array}{l}\text { La planeación sí ha servido para } \\
\text { mejorarla }\end{array}$ & 78 & $94 \%$ \\
\hline & $\begin{array}{l}\text { La planeación no ha servido } \\
\text { para mejorarla }\end{array}$ & 5 & $6 \%$ \\
\hline \multirow[b]{2}{*}{ Tipo de IES } & Universidad & 37 & $45 \%$ \\
\hline & $\begin{array}{l}\text { Otra (Institución Universitaria/ } \\
\text { Escuela Tecnológica, Institución } \\
\text { Tecnológica, Institución Técnica } \\
\text { Profesional) }\end{array}$ & 46 & $55 \%$ \\
\hline \multirow{2}{*}{$\begin{array}{l}\text { Estado del Plan } \\
\text { Estratégico }\end{array}$} & Plenamente en operación & 75 & $90 \%$ \\
\hline & $\begin{array}{l}\text { Otra (en elaboración, pendiente } \\
\text { de iniciar implementación) }\end{array}$ & 8 & $10 \%$ \\
\hline
\end{tabular}

Fuente: elaboración propia.

La distribución porcentual de las instituciones de educación superior en Colombia, según carácter, es de $56 \%$ de instituciones pública, y $44 \%$ de instituciones privadas.

Para determinar cuál es el efecto que tiene el seguimiento a la gestión estratégica sobre las tres funciones sustantivas de las IES, esta investigación hace uso de modelos de regresión binaria tipo Probit, que nos permita evaluar si hay diferencia significativa en la mejoría de las funciones sustantivas al realizar seguimiento a la gestión estratégica.

Partiendo de lo anterior, se realizan tres regresiones con modelos tipo Probit, que utilizan como variables dependientes las tres funciones sustantivas, docencia, investigación y extensión; que toman el valor de 1 si se ha presentado mejoría y 0 en el caso contrario. Las funciones sustantivas van a ser explicadas por tres variables independientes, el seguimiento a la gestión estratégica se toma como el promedio entre las variables monitoreo y evaluación, resultados de autoevaluación, sistemas de indicadores y la revisión de unidades internas. Con dicha variable se busca apreciar la dirección del efecto sobre las funciones sustantivas y las dos siguientes de tipo control como lo son el tipo de universidad y el estado actual del plan estratégico. Las variables de control toman los valores de 1 cuando es Institución Universitaria, Institución Tecnológica o Institución Técnica, y 0 cuando es Universidad, y el estado actual del plan estratégico toma los valores de 1 si el plan está plenamente en operación y 0 si no ha empezado operación o se encuentra en elaboración.

Se proponen los siguientes modelos y se estiman por un modelo de probabilidad tipo Probit: 


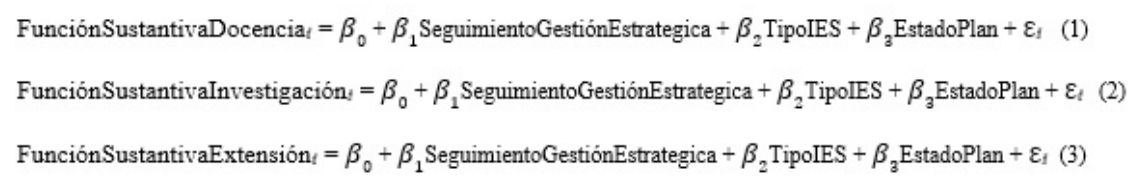

Para facilitar la interpretación se reportan los coeficientes estimados del modelo Probit, se presenta la tabla 8.

TABLA 8

Análisis multivariante

\begin{tabular}{|c|c|c|c|}
\hline \multirow{2}{*}{$\begin{array}{c}\text { Variables } \\
\text { Independientes }\end{array}$} & (1) & (2) & (3) \\
\hline & Docencia & Investigación & Extensión \\
\hline $\begin{array}{l}\text { Seguimiento a la } \\
\text { gestión estratégica }\end{array}$ & $\begin{array}{r}1,92^{* * *} \\
(0,65)\end{array}$ & $\begin{array}{l}1,57 * * \\
(0,69)\end{array}$ & $\begin{array}{l}1,21^{*} \\
(0,70)\end{array}$ \\
\hline Tipo de IES & $\begin{array}{r}0,07 \\
(0,48)\end{array}$ & $\begin{array}{r}-0,17 \\
(0,46)\end{array}$ & $\begin{array}{l}-0,77^{*} \\
(0,45)\end{array}$ \\
\hline $\begin{array}{l}\text { Estado del Plan } \\
\text { Estratégico }\end{array}$ & $\begin{array}{r}0,52 \\
(0,52)\end{array}$ & $\begin{array}{r}0,51 \\
(0,52)\end{array}$ & $\begin{array}{l}0,95^{*} \\
(0,53)\end{array}$ \\
\hline Constante & $\begin{array}{r}-0,53 \\
(0,70)\end{array}$ & $\begin{array}{r}-0,21 \\
(0,73)\end{array}$ & $\begin{array}{r}0,27 \\
(0,86)\end{array}$ \\
\hline $\begin{array}{l}\text { Número de } \\
\text { observaciones }\end{array}$ & 83 & 83 & 83 \\
\hline Pseudo $R^{2}$ & 0,21 & 0,15 & 0,21 \\
\hline Wald Chi2 & 11,39 & 7,87 & 15,99 \\
\hline Prob $>$ Chi 2 & 0,00 & 0,04 & 0,00 \\
\hline
\end{tabular}

Nota: errores estándar entre paréntesis. $\left({ }^{*}\right): \mathrm{p}<0,10 ;\left({ }^{* *}\right): \mathrm{p}<0,05 ;\left({ }^{* * *}\right): \mathrm{p}<0,01$

Fuente: elaboración propia.

A diferencia de los modelos lineales, el efecto que tiene las variables explicativas sobre la probabilidad de que las funciones sustantivas de probabilidad hayan mejorado no es independiente del vector de características Xi, es decir, la manera en la que las variables independientes impactan la probabilidad de mejoría en las funciones sustantivas no sólo depende de los coeficientes sino también del valor tomarían las variables. Por lo cual, cambia la manera como el investigador se acerca a la interpretación de los datos, se debería realizar el cálculo de los efectos marginales para hacer las interpretaciones. En suma, el propósito de la investigación es constatar si el seguimiento ha surtido un efecto positivo por lo que se analizará la significancia de la variable seguimiento a la gestión estratégica en las tres funciones sustantivas y la dirección o sentido más que el efecto. Sin embargo, en la tabla 9 se presentan los efectos marginales.

TABLA 9

Efectos marginales de las funciones sustantivas

\begin{tabular}{|l|c|c|c|}
\hline \multirow{2}{*}{$\begin{array}{l}\text { Variables } \\
\text { Independientes }\end{array}$} & $(1)$ & $(2)$ & $(3)$ \\
\cline { 2 - 4 } & Docencia & Investigación & Extensión \\
\hline $\begin{array}{l}\text { Seguimiento a la } \\
\text { Gestión Estratégica }\end{array}$ & 0,18 & 0,18 & 0,11 \\
\hline Tipo de IES & $(0,08)$ & $(0,09)$ & $(0,06)$ \\
\hline $\begin{array}{l}\text { Estado del Plan } \\
\text { Estratégico }\end{array}$ & 0,00 & $-0,02$ & $-0,07$ \\
$(0,04)$ & $(0,05)$ & $(0,05)$ \\
\hline $\begin{array}{l}\text { Número de } \\
\text { observaciones }\end{array}$ & 0,04 & 0,05 & 0,08 \\
& $(0,04)$ & $(0,05)$ & $(0,05)$ \\
\hline
\end{tabular}

Nota: errores estándar entre paréntesis. Fuente: elaboración propia

Considerando los resultados presentados en la tabla 9 se puede afirmar que las funciones sustantivas se ven impactadas de forma positiva por el seguimiento a la gestión estratégica, y además al realizar el análisis para niveles de significancia, la docencia se impacta con un nivel significancia del $1 \%(\mathrm{H}$.), mientras que la 
investigación es del 5\% (H.) y la extensión es del 10\% (H.). Se podría inferir que con un nivel de confianza de $99 \%$ el seguimiento a la gestión estratégica surte un efecto positivo en la función sustantiva de docencia. Adicionalmente, con un nivel de confianza de $95 \%$ se infiere que el seguimiento a la gestión estratégica surte un efecto positivo en la función sustantiva de investigación, y, por último, con un nivel de confianza del 90\% se infiere que el seguimiento a la gestión estratégica tiene un efecto positivo sobre la función sustantiva de extensión.

El efecto marginal del Seguimiento a la Gestión Estratégica en las funciones sustantivas de Docencia e Investigación es del 18\%, es decir, el seguimiento a la gestión estratégica aumenta en un 18\% la función sustantiva de docencia y de investigación. Por otra parte, el seguimiento a la gestión estratégica genera un aumento del $11 \%$ en la función sustantiva de extensión. Lo anterior indica que el seguimiento a la gestión estratégica tiene un mayor impacto en las funciones sustantivas de docencia e investigación y que este efecto puede llegar a ser el mismo.

Por otra parte, al considerar el tipo de institución educativa en el caso de que la institución sea universitaria, tecnológica o técnica la función sustantiva de investigación disminuye en un $2 \%$, mientras que, la función sustantiva de extensión en un 7\%. Para la función sustantiva de docencia el efecto es mínimo. Finalmente, los efectos marginales del estado del plan estratégico indican que si el plan se encuentra en operación aumenta en un $4 \%$ la función sustantiva de docencia, en el caso de la función sustantiva de investigación este aumento es del $5 \%$ y para el caso de la función sustantiva de extensión es del $8 \%$. Lo anterior indica que la magnitud del efecto cuando el plan estratégico se encuentra en operación es mayor para la función sustantiva de extensión.

De acuerdo con los planteamientos de Jiménez (2006), la proyección social de las instituciones tiene codependencia al nivel de desarrollo de la implementación de sus programas de investigación. Botero citado en Rico Molano (2016)-, reafirma esta teoría, resaltando que la gestión educativa tiene tendencias hacia la autonomía, la democratización y la calidad. Cabe destacar que la democratización de la gestión educativa incluye su interacción con la sociedad, mientras que la calidad de la educación abarca la precisión con la que esta pueda darle resolución a las problemáticas sociales. Por lo tanto, el autor sugiere la necesidad de formar y actualizar constantemente a la docencia, por medio de herramientas como la investigación. Tales herramientas pueden estar dirigidas según sus acciones estratégicas, como por ejemplo la creación de programas de maestría, o el enfoque investigativo, que en instituciones privadas pueden tener un fin comercial, mientras que en aquellas instituciones que son de carácter público puede tener un fin social. Por tal razón, no se descarta que con una mayor tasa de respuesta se lograse reafirmar o refutar que las instituciones universitarias, instituciones tecnológicas e instituciones técnicas tienen menor probabilidad de desarrollar la función sustantiva de extensión con respecto a las Universidades.

El modelo también indica que en la medida que las IES tengan el plan estratégico en plena operación se presenta mayor probabilidad de mejoría en la función sustantiva de extensión. Esto puede estar directamente relacionado con las fases de la administración estratégica, reducidas por Velandia y Girotto (2015) a cuatro estaciones: el pensar la estrategia, la ejecución de acciones para alcanzar los objetivos planteados, y dos fases de aprendizaje que distinguen la evaluación y monitoreo de los procedimientos que se realizaron, y las transformaciones requeridas en asignación y usos de recursos para incrementar los resultados positivos de esos resultados. Para citar sólo un ejemplo: una de estas fases (la ejecución de acciones) puede ocupar un espacio temporal prolongado, como consecuencia de tener que cumplir las disposiciones legales, como la Ley 30 (1992), Ley 749 (2002), Ley 115 (1994) o Decreto Ley 2150 (1995) que, entre otros, determina todas aquellas disposiciones que deben cumplir las IES. Por lo cual se debe tomar en cuenta el estado actual del plan estratégico como un factor significativo en el desarrollo de las funciones sustantivas.

Teniendo en cuenta el tamaño de la muestra, el modelo no presenta relación significativa entre el tipo de institución y el estado actual del plan estratégico con las funciones sustantivas de docencia e investigación. La investigación presente parte de la idea de que la administración estratégica en los distintos tipos de IES presenta un grado de homogeneidad, lo cual puede ser respaldado como consecuencia de que autores como 
Kniola (2013), Keller (2007) y De Waal y Kerklaan (2015), tratan sus investigaciones y casos de estudio sin discriminar el tipo de institución.

\section{Discusiones y consideraciones}

En la investigación que fue dirigida por la red Telescopi Colombia, el equipo buscó determinar si existía una relación de incidencia entre cuatro variables independientes del seguimiento a la gestión estratégica en las IES sobre tres de sus funciones sustantivas (docencia, investigación y extensión); con el tipo de IES y el estado actual del plan estratégico como variables de control, la relación que se planteó como hipótesis, partiendo de una investigación ardua de literatura, fue que el seguimiento en la gestión estratégica tiene una dirección positiva en las funciones sustantivas.

La investigación, como resultado del estudio cuantitativo realizado con el método Probit, y apoyado con la información que fue descubierta en otras investigaciones, y que se incluyeron en el marco teórico, arrojó que, efectivamente, el seguimiento a la gestión estratégica tenía un impacto positivo en las tres funciones sustantivas. Adicionalmente, la investigación teórica de las variables permite plantear dos dilemas importantes que podrían ser analizados a mayor profundidad en futuros estudios: El primer dilema consiste en la existencia de una interrelación de las variables dependientes, pues según González y Arcángel (2013), la investigación y la docencia son dependientes entre ellas y son simultáneamente dependientes de la proyección social de las IES; por otra parte, los resultados ponen en consideración que la valoración de cada IES sobre sus propios objetivos estratégicos, determinara la forma en que se evalúan, monitorean y miden las tareas que les permitirá alcanzar esos objetivos.

Los resultados que arroja la aplicación de la herramienta Probit permitió determinar que las funciones elegidas fueron impactadas por el Seguimiento a la Gestión Estratégica Universitaria -SGEU- en un nivel mayor a menor en el siguiente orden: docencia, investigación y extensión; claramente analizados en términos de significancia, comprobando así las tres hipótesis planteadas. Sin embargo, no fue posible encontrar literatura que pudiese sustentar la razón por la cual las funciones sustantivas de investigación y extensión se pudieran ver impactadas en un menor nivel por las variables independientes del seguimiento al plan estratégico, en comparación con el impacto en la función docencia.

Lo anterior es importante porque se abre la puerta a la investigación en el sentido que, si bien el estudio se basó en el análisis direccional del efecto; es decir, en el observar que un seguimiento compuesto por el monitoreo y la evaluación, por los resultados de autoevaluación, por el sistema de indicadores y por la revisión en unidades internas, produce un efecto positivo en las funciones sustantivas; el resultado positivo permite inclinar nuevos análisis, por ejemplo a qué otros factores pueden ser parte de la variable de seguimiento a la gestión estratégica que aumenten la probabilidad de mejoría en las funciones sustantivas, también se podría realizar un análisis interno de por qué las funciones sustantivas de investigación y extensión se ven impactadas en menor manera o por qué son menos significativas.

Lo expuesto podría ser considerado teniendo en cuenta que la extensa caracterización interna y externa de las IES fue reducida a la investigación sobre la incidencia de cuatro variables independientes, sobre tres variables dependientes, cuyo punto de partida es la apreciación de un solo actor institucional: la dirección universitaria. Sin embargo, el estudio de la red Telescopi en 2011 mostraba que el seguimiento a la gestión estratégica era lo más importante y relevante a reconocer a la dirección universitaria, lo cual abre un camino de posibilidades donde, según los contextos, se puedan agregar más factores incidentes en las funciones sustantivas, siempre y cuando exista un mayor alcance en términos de datos, que podrían implicar un estudio más amplio, con un mayor número de variables independientes y variables de control, pero, sobre todo, con muestras que permitan una participación más amplia de los actores participantes en el SGEU. 
Por lo tanto, en primera instancia, sería incorrecto afirmar que no existen otras características de las IES que también serían impactadas por las variables independientes y, por el contrario, que otras variables del plan estratégico de las IES tuviesen un mayor impacto en las funciones sustantivas de lo que se pudo determinar para aquellas que constituían el seguimiento del plan estratégico. También es claro que el análisis se enriquece en tanto se pueda medir desde varios frentes; es decir, con la inclusión de otras variables al modelo.

Finalmente, se debe destacar que el modelo Probit fue el más acertado para los propósitos de la investigación, que pretende categorizar si existía un impacto en la probabilidad de mejoría en las funciones sustantivas, sabiendo que la importancia de la investigación recae en encontrar la dirección más que el efecto. Adicionalmente, este modelo es una herramienta útil para un análisis estadístico de múltiples variables y su incidencia en el resultado final de aquello que se indaga.

\section{Conclusiones}

El resultado más significativo de esta investigación es la relación de impacto entre el seguimiento a la gestión estratégica sobre la función sustantiva de docencia, lo cual apoya la primera hipótesis ( $H 1)$. En este sentido, la función docencia debe conducir al desarrollo de conocimientos, habilidades y destrezas en las personas; sin embargo, teniendo en cuenta que la información que permite el desarrollo no es estática, sino cambiante, en la medida en que el plan estratégico se encuentre bajo seguimiento será posible determinar si las acciones que cobijan la función docencia realmente satisfacen las necesidades y vacíos actuales de los entornos a los cuales quieren responder las universidades.

Por otra parte, el modelo Probit demostró que existe una relación de impacto entre el seguimiento a la gestión estratégica y la función sustantiva de investigación (H2), sustentado en que toda IES ofrecen servicios académicos que deben partir de un saber que pueda ser debidamente aplicado en sus respectivos campos de acción y, por lo tanto, cada uno de los programas académicos que ofrecen se deben estar ajustados a la realidad contextual, lo cual implica que las IES formen a sus docentes en las necesidades de sus respectivos campos, por medio de herramientas tales como la investigación.

Pese a que se logró comprobar que existe una relación de incidencia entre el seguimiento a la gestión estratégica y la función sustantiva de extensión (H3), este fue el que demostró ser impactado en un menor grado por la gestión estratégica. Aún no existen suficientes investigaciones que puedan afirmar la razón por la cual se presenta tal relación, pero algunos investigadores como Velandia y Girotto (2015) plantean que la función de extensión se ve impactada positivamente por las cuatro fases de la administración estratégica.

Los resultados permiten inferir que el seguimiento a la gestión estratégica va a permitir aumentar la productividad, efectividad, eficiencia, de la docencia, la investigación y la extensión de las Instituciones de Educación Superior -IES-, por lo cual es prioritario que las IES enfoquen una atención ardua al componente, puesto que es tendencia que un aumento en la mejoría de dichas funciones sustantivas se podría traducir en aumentos de calidad en la educación, en los productos de investigación y en la estructura la extensión.

A continuación, se plantean diversos factores que podrían evidenciar niveles de significancia mejores y un análisis más enriquecedor que los obtenidos en este estudio y que se constituyen en oportunidades de investigaciones futuras:

- No hay suficiente evidencia en la literatura donde se utilicen análisis cuantitativos para explorar la tesis de investigación, se deberían consolidar más datos, con mayor alcance para tratar de explicar con más variables el fenómeno de estudio, lo que ayudaría no sólo a fundamentar más la dirección positiva del seguimiento hacia las funciones sustantivas sino a caracterizar la magnitud del efecto.

- Se recomienda no solo ampliar el tamaño de la muestra, sino también permitir que otros directivos, adicionales a las rectorías, participen del diligenciamiento de la encuesta. Esto permitirá reducir el sesgo generado por dar una respuesta sobre la propia gestión de los encuestados. 
- Se recomienda indagar, utilizar y desplegar las herramientas pertinentes que permitan en investigaciones futuras obtener una mayor tasa de respuesta de los participantes, con el fin de ofrecer a los lectores resultados que generen confianza y respaldo de su parte.

- En la investigación se procuró realizar un marco teórico sólido con el cual se pudo explorar un poco más el concepto de seguimiento a la gestión estratégica, y con estos hallazgos en literatura consolidar el método.

- Para futuras investigaciones, se insta a investigar la relación de incidencia de las características contextuales de las IES, sobre las variables dependientes e independientes de la presente investigación, y que puedan afectar el resultado final de la misma.

En resumen, el presente estudio alcanzó el objetivo propuesto: comprobar si el Seguimiento a la Gestión Estratégica Universitaria generaba algún efecto en el desempeño de las tres funciones sustantivas que fueron seleccionadas para la investigación. El resultado que se obtuvo se sustenta matemáticamente al utilizar como herramienta el método estadístico Probit, el cual arrojó datos que permiten concluir que hay una relación entre las variables dependientes e independientes. Sin embargo, se debe mencionar que el presente estudio también está compuesto por un alto nivel de investigación teórico, que no sólo apoya los resultados estadísticos, sino que también funge como una explicación lógica de las razones por las que se presentaron los resultados obtenidos.

\section{Referencias}

Alonso M., P. (2019). El perfil del buen docente universitario según la valoración de alumnos de Magisterio y Psicopedagogía. Perfiles Educativos, 41(164), 68-81. https://doi.org/10.22201/iisue.24486167e.2019.164.589 06

Álvarez, J. D., Tortosa, M. T., \& Pellín, N. (2015). Investigación y propuestas innovadoras de redes UA para la mejora docente. Documento de trabajo. Universidad de Alicante, 1428-1443. http://rua.ua.es/dspace/bitstream/10045/44926/2/Investigaci\%c3\%b3n\%20y\%20Propuestas\%2 OInnovadoras\%20de\%20Redes\%20UA\%20para\%20la\%20Mejora\%20Docente.pdf

Aspromourgos, T. (2012). The Managerialist University: An Economic Interpretation. Australian Universities'Review, 54(2), 44-49. https://files.eric.ed.gov/fulltext/EJ981193.pdf

Broadbent, J., \& Laughlin, R. (2009). Performance management systems: A conceptual model. Management Accounting Research, 20(4), 283-295. https://doi.org/10.1016/j.mar.2009.07.004

Broadbent, J., Gallop, C., \& Laughlin, R. (2010). Analysing societal regulatory control systems with specific reference to higher education in England. Accounting, Auditing \& Accountability Journal, 23(4), 506-531. https://doi.or g/10.1108/09513571011041606

Cáceres, C. (2007). Planeación estratégica en universidades del Consejo de Rectores: evidencias del periodo 2000-2005. Calidad en la educación, 27, 108-150. https://doi.org/10.31619/caledu.n27.221

Canales, A. (2011). El dilema de la investigación. Perfiles Educativos, XXXIII(número especial), 34-44. http://www. scielo.org.mx/pdf/peredu/v33nspe/v33nspea4.pdf

Centro Interuniversitario de Desarrollo. (1992).Administración Universitaria en América Latina. Una perspectiva estratégica. Santiago: Centro Interuniversitario de Desarrollo.

Centro Interuniversitario de Desarrollo (1999). Manual Gestión Estratégica Universitaria: Procesos Administrativos y Financieros Programa. Programa De Intercambio Universitario entre la Unión Europea y América Latina. Santiago: Alfa.

Centro Interuniversitario de Desarrollo (2007). Acreditación y Dirección Estratégica para la Calidad en las Universidades. Santiago: Centro Interuniversitario de Desarrollo. 
Chenhall, R. H. (2003). Management control systems design within its organizational context: Findings from contingency-based research and directions for the future. Accounting, organizations and society, 28(2), 127-168. https://doi.org/10.1016/S0361-3682(01)00027-7

Chenhall, R. H., \& Euske, K. J. (2007). The role of management control systems in planned organizational change: An analysis of two organizations. Accounting, Organizations and Society, 32(7), 601-637. https://doi.org/10.1 016/j.aos.2006.09.007

Chenhall, R. H., Hall, M., \& Smith, D. (2010). Social capital and management control systems: A study of a nongovernment organization. Accounting, Organizations and Society, 35(8), 737-756. https://doi.org/10.1016/j.ao s.2010.09.006

Concepción P., N. S., \& Trujillo S., Z. de la C. (2014). La extensión universitaria como vía para fortalecer el trabajo educativo en la UCM de Pinar del Río. Revista de Ciencias Médicas de Pinar del Río, 18(5), 852-862. http://sci elo.sld.cu/scielo.php?script=sci_arttext\&pid=S1561-31942014000500014\&lng=es\&tlng=es.

Cook, C., Heath, F., \& Thompson, R. L. (2000). A meta-analysis of response rates in web-or internet-based surveys. Educational and Psychological Measurement, 60(6), 821-836. https://doi.org/10.1177/00131640021970934

Cook, D. C., Nelson, E.-L., Ast, C., \& Lillis, T. (2013). A Systematic Strategic Planning Process Focused on Improved Community Engagement by an Academic Health Center: The University of Kansas Medical Center's Story. Academic Medicine\#: Journal of the Association of American Medical Colleges, 88(5), 1-6. https://doi.org/10.10 97/ACM.0b013e31828a3ba4

De Dios-Alija, T., García Ramos, J.M., \& Muñoz-Pérez, S. (2017). Diseño de un modelo de evaluación y desarrollo docente en una universidad privada. Revista Complutense de Educación, 28(1), 61-80. https://doi.org/10.5209 /rev_RCED.2017.v28.n1.48718

De Waal, A., \& Kerklaan, L. (2015). Developing an evidence-based management approach for creating highperformance in higher educational institutions. Academy of Educational Leadership Journal, 19(3), 83-101. htt ps://www.abacademies.org/articles/AELJ_Vol_19_No_3_2015.pdf

Decreto Ley 2150. Ministerio de Educación Nacional [En línea], Bogotá, Colombia, 5 de diciembre de 1995

Deem, R. (1998). 'New managerialism' and higher education: The management of performances and cultures in universities in the United Kingdom. International Studies in Sociology of Education, 8(1), 47-70. https://doi.or $\mathrm{g} / 10.1080 / 0962021980020014$

Deem, R., \& Brehony, K. J. (2005). Management as ideology: The case of 'new managerialism' in higher education. Oxford Review of Education, 31(2), 217-235. https://doi.org/10.1080/03054980500117827

Del Huerto, M. E. (2007). La Extensión Universitaria como vía para fortalecer los vínculos Universidad-Sociedad desde la promoción de salud. Revista Cubana de Salud Pública, 33(2) Recuperado en 17 de octubre de 2018, de http:/ /scielo.sld.cu/scielo.php?script=sci_arttext\&pid=S0864-34662007000200005\&lng=es\&tlng=es

García, A., \& Palomares, D. (2009). Examining benchmark indicator systems for the evaluation of higher education institutions. Higher Education: The International Journal of Higher Education Research, 60, 217-234. https://d oi.org/10.1007/s10734-009-9296-8

González R., O., \& Arcángel U., R. (2013). Investigación Universitaria Herramienta de Transformación Social. Formación Gerencial, 12(2), 193-210. https://dialnet.unirioja.es/descarga/articulo/4772669.pdf

Ferreira, A., \& Otley, D. (2009). The design and use of performance management systems: An extended framework for analysis. Management Accounting Research, 20(4), 263-282. https://doi.org/10.1016/j.mar.2009.07.003

Jiménez, W. G. (2006). La formación investigativa y los procesos de investigación científico-tecnológica en la Universidad Católica de Colombia. Studiositas, 1(1), 45-52. https://repository.ucatolica.edu.co/bitstream/109 83/474/1/Stud_1-1_A06_Formaci\%c3\%b3n\%20investigativa.pdf

Keller, G. (2007). Higher education management: Challenges and strategies. En J. J. Forest \& P. G. Altbach, International Handbook of Higher Education (pp. 229-242). Dordrecht: Springer.

Kniola, D. J. (2013). Accountability through assessment of administrative organizations in higher education. European Journal of Higher Education, 3(1), 89-101. https://doi.org/10.1080/21568235.2013.778044 
Larios Gómez, E. (2015). La gestión educativa para la Universidad pública y privada en México: una comparación competitiva. Red Internacional de Investigadores en Competitividad, 9(1). 1589-1606. https://www.riico.net/in dex.php/riico/article/view/89/216

Ley 115 (1994). Ministerio de Educación Nacional [En línea], Bogotá, Colombia, 8 de febrero de 1994.

Ley 749 (2002). Ministerio de Educación Nacional [En línea], Bogotá, Colombia, 19 de julio de 2002.

Ley 30 (1992). Ministerio de Educación Nacional [En línea], Bogotá, Colombia, 28 de diciembre de 1992.

López, C., Sánchez, Á., Pardo, R., \& Zapata, S. (2017). Modelo de gestión de proyectos de la Universidad EAFIT aplicados al sistema general de regalías en ciencia, tecnología e innovación. Revista Ciencias Estratégicas, 24(36), 271-289. http://dx.doi.org/10.18566/rces.v24n36.a2

Llinàs-Audet, X., Girotto, M., \& Parellada, F. S. (2011). La dirección estratégica universitaria y la eficacia de las herramientas de gestión: el caso de las universidades españolas. Revista de Educación, 355(1), 33-54. https://se de.educacion.gob.es/publiventa/d/22862/19/1

Ministerio de Educación Nacional - MEN (2007). Glosario de la Educación Superior. Bogota, Colombia. Ministerio de Educación Nacional. https://www.mineducacion.gov.co/sistemasdeinformacion/1735/articles-213912_glo sario.pdf

Meyer Junior, V., Pascuci, L. M., \& Meyer, B. (2018). Strategies in Universities: tensions between macro intentions and micro actions. Journal of Contemporary Administration, 22(2), 163-177. https://doi.org/10.1590/1982-7 849 rac2018160081

Morán O., P. (2004). La docencia como recreación y construcción del conocimiento Sentido pedagógico de la investigación en el aula. Perfiles educativos, 26(105-106), 41-72. http://www.scielo.org.mx/scielo.php?script=sc i_arttext\&pid=S0185-26982004000100003\&lng=es\&tlng=es.

Murias, P., de Miguel, J. C., \& Rodríguez, D. (2007). A composite indicator for university quality assessment: The case of Spanish Higher Education System. Social Indicators Research, 89, 129-146. https://doi.org/10.1007/s11205 $-007-9226-\mathrm{z}$

Navas, L. P., Montes, F., Abolghasem, S., Salas, R. J., Toloo, M., \& Zarama, R. (2020). Colombian higher education institutions evaluation. Socio-Economic Planning Sciences, 71. https://doi.org/10.1016/j.seps.2020.100801

Otley, D. (2003). Management control and performance management: Whence and whither? The British Accounting Review, 35(4), 309-326. https://doi.org/10.1016/j.bar.2003.08.002

Parakhina, V., Godina, O., Boris, O., \& Ushvitsky, L. (2017). Strategic management in universities as a factor of their global competitiveness. International Journal of Educational Management, 31(1), 62-75. http://dx.doi.org/10. 1108/ijem-03-2016-0053

Parker, L. D. (2002). It's been a pleasure doing business with you: a strategic analysis and critique of university change management. Critical Perspectives on Accounting, 13(5-6), 603-619. https://doi.org/10.1006/cpac.2002.0561

Parker, L. D. (2013). Contemporary university strategising: the financial imperative. Financial Accountability \& Management, 29(1), 1-25. https://doi.org/10.1111/faam.12000

Ramírez- Gutiérrez, Z., Barrachina-Palanca, M., \& Ripoll-Feliu, V. (2020) Efficiency in higher education. Empirical study in public universities of Colombia and Spain. Revista de Administração Pública, 54(3), 468-500. https:// doi.org/10.1590/0034-761220190232x

Rico Molano, A. D. (2016). La gestión educativa: hacia la optimización de la formación docente en la educación superior en Colombia. Sophia, 55-70. http://www.scielo.org.co/pdf/sph/v12n1/v12n1a04.pdf

Rivero, S., Diaz, M., López, M. J., \& Rodríguez, R. J. (2018). Indicator system for managing science, technology and innovation in universities. Scientometrics, 115, 1575-1587. https://doi.org/10.1007/s11192-018-2721-y

Sánchez-del-Arco, P., \& Peón, P. (2017). Inteligencia institucional en la Universidad Nacional de Educación a Distancia, UNED: Modelo de indicadores clave institucionales (KPIs) Business Intelligence in Universidad Nacional de Educación a Distancia, UNED: Model of Key Performance Indicators (KPI. La Cuestión Universitaria, 9(1), 184-199. http://polired.upm.es/index.php/lacuestionuniversitaria/article/viewFile/3581/ 3663 
Shah, M., \& Nair, S. (2011). The influence of Strategy and External Quality Audit on University Performance: An Australian perspective. Tertiary Education And Management, 17(2), 139-150. https://doi.org/10.1080/13583 883.2011.557778

Universidad del Bosque (2014). Politica de Proyección Social y Responsabilidad Universitaria. Bogotá: Universidad del Bosque. https://www.unbosque.edu.co/sites/default/files/2017-06/politica_proyeccion_responsabilidad_soci al_universitaria.pdf

Universidad del Valle (2012). Proyección Social y Extensión. Cali, Colombia. Universidad del Valle. https://www.uni valle.edu.co/proyeccion-social

Universidad Nacional de Colombia (2005). Por el cual se adopta el Estatuto General de la Universidad Nacional de Colombia. Bogotá, Colombia. Universidad Nacional de Colombia. http://www.legal.unal.edu.co/rlunal/home /doc.jsp?d_i=35137

Velandia, C., \& Girotto, M. (2015). Strategic Management in Universities: A conceptual framework based on IberoAmerican higher education systems. In J. Cifuentes-Madrid et al. (eds.), Strategic Management of Universities in the Ibero-America Region (pp. 1-43). Cham: Springer International Publishing.

Willmott, H. (1995). Managing the Academics: Commodification and Control in the development of university education in the UK. Human relations, 48(9), 993-1027. https://doi.org/10.1177/001872679504800902

\section{Notas}

* Artículo de investigación.

\section{Licencia Creative Commons CC BY 4.0}

Para citar este artículo: González-Campo, C. H., Vásquez-Rivera, O. I., \& Cifuentes-Madrid, J. (2020). Efecto del seguimiento a la gestión estratégica sobre las funciones sustantivas en las Instituciones de Educación Superior en Colombia. Cuadernos de Administración, 33(61). https://doi.org/10.11144/Javeriana.cao33.e sge 